\title{
Schizophrenie als Leitkrankheit der Psychiatrie
}

\author{
Schizophrenia as the Prototypical Psychiatric Disorder
}

Autoren

Pro: $\quad$ Roland Vauth

Kontra: Fritz Hohagen, Winfried Lotz-Rambaldi

Bibliografie

DOI http://dx.doi.org/

10.1055/s-0032-1304920

Psychiat Prax 2012; 39 :

153-156

(c) Georg Thieme Verlag KG

Stuttgart · New York

ISSN 0303-4259

Korrespondenzadressen

PD Dr. med. Dipl.-Psych.

Roland Vauth

Psychiatrische

Universitätspoliklinik,

Universitäre Psychiatrische

Kliniken

Claragraben 95

CH-4005 Basel

roland.vauth@upkbs.ch

Prof. Dr. Fritz Hohagen Dipl.-Psych. Winfried Lotz-Rambaldi

Zentrum für Integrative Psychiatrie ZIP gGmbH Ratzeburger Allee 160

23538 Lübeck

winfried.lotz-rambaldi@uksh.de

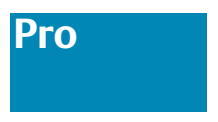

Von Leitkrankheiten in der Medizin erwartet man, dass sie Positionierung und ggf. Umorientierung eines Faches bestimmen. Wohl kaum eine Erkrankung aus dem Spektrum psychischer Erkrankungen hat die Psychiatrie und Psychotherapie nach innen und nach außen in dermaßen starker Weise geprägt wie die Schizophrenie.

Andererseits wurde für die Behandlung schizophrener Erkrankungen viel aus der Versorgung anderer Störungen gelernt. Die für diesen Beitrag aufgetragene Zuspitzung auf eine pointierte, dichotom vereinfachende Position zwingt jedoch dazu, sich auf einige richtungsweisende Aspekte der Auseinandersetzung unseres Faches mit schizophrenen Störungen in Forschung und Praxis zu beschränken, um sie als Leiterkrankung - im schon Errungenen, aber auch im dringend Nachzuholenden - zu „verteidigen“. Welche Errungenschaften im Verstehen und Behandeln schizophrener Störungen können als Orientierungslinie unseres gesamten Faches gelten?

Argument 1: Die biopsychosoziale Ursachen- und Interventionsforschung zu schizophrenen Störungen spielte eine Vorreiterrolle bei der Überwindung des Leib-Seele-Dualismus in unserem Fach. Das „biopsychosoziale“ Modell und das „Vulnerabilitäts-Stress-Kompetenz-Modell“ [1] bringen als ätiologische Konzepte für unser Fach auf beispielhafte Weise die Vielfalt der Erkenntnisse moderner molekularbiologischer, genetischer, Bildgebungs-, epidemiologischer und psychologischer Befunde zugunsten eines multidimensionalen Ursachenmodells zusammen. Dabei wird die dopaminerge Dysregulation verstanden als „final common pathway“ multipler genetischer, intrauteriner und umgebungsbezogener „Noxen“. Dekompensation wird als schrittweiser Prozess betrachtet und als Folge eines Ungleichgewichts protektiver und belastender Faktoren. Nicht nur für die Ätiologie, sondern auch für Verlauf, Prognose und Therapie sind multidimensionale Konzepte bei schizophrenen Störungen breit akzeptiert. Neben der Pharmakotherapie erwiesen sich bspw. auch multiple psychologische Faktoren (Zielorientierung, Erfolgszuversicht, Beibehalt von Substanzabstinenz usw.) als entscheidend [2], die sich verhaltenstherapeutisch fokussieren lassen $[3,4]$.

Bis weit in die 90er-Jahre hinein war die Dichotomie „endogener“ versus „neurotischer“ Störungen Grundlage differenzialtherapeutischer Entscheidungen unseres Fachs. Psychotherapie war zuvor ausschließlich für „neurotische“ oder „reaktive“ Störungen indiziert, die dann selbst bei schweren Syndromen keine Psychopharmakotherapie erhielten. Psychotherapie bei „endogenen Störungen“ - zuvorderst bei der Schizophrenie wurde als symptomverschlimmernd und rückfallfördernd befürchtet und daher großteils als kontraindiziert betrachtet. „Endogene Störungen“ waren meist die Domäne ausschließlicher Psychopharmakologie. Erst der Nachweis neurobiologischer Veränderungen durch Psychotherapie auch bei „endogenen Störungen“ [5, 6] - sowie die Entwicklung und Wirksamkeitsbelege über rein edukatives Arbeiten hinausgehender, störungsspezifischer, zumeist verhaltenstherapeutischer, Verfahren [7] brachte die Wende.

Argument 2: Die Überwindung des Mythos vom qualitativ andersartigen psychotischen Erleben war eine der Voraussetzungen für das Nutzbarmachen wirksamer psychologischer Konzepte zu Verständnis und Behandlung schizophrener Symptomatik.

Das scheinbar qualitativ andersartige psychotische Erleben im Vergleich zu normalpsychologischen Prozessen sozialer Wahrnehmung und die hieraus resultierende scheinbare Unbegreiflichkeit haben von außen Stigmatisierung (das Anderssein, das Ver-Rückte, das fremd und bedrohlich ist), aber auch nach innen das Herantragen normalpsychologischer Erkenntnisse an Verständnis und Psychotherapie über Jahrzehnte ver- 
hindert $[8,9]$. So war die Pionierarbeit über kognitions- und sozialpsychologische Prozesse bei wahnhafter Symptomatik von Aaron Beck bereits nahezu 4 Jahrzehnte bekannt , bevor kognitionsund sozialpsychologische Grundlagenforschung in die Entwicklung evidenzbasierter kognitiv-behavioraler Interventionen mündete $[10,11]$.

Argument 3: Die Überwindung des Mythos vom qualitativ Andersartigen war Einstieg in erste Schritte zur Überwindung von Stigmatisierung [12] und Selbststigmatisierung [13] und trug damit zur Überwindung der Gefährdung nachhaltiger Therapiebindung bei.

Die Sorge von Menschen mit schizophrenen Störungen vor sozialem Ausschluss z.B. am Arbeitsplatz, im Freundes- und Bekanntenkreis, ist nicht nur bei psychopathologisch weitgehend stabilen Patienten hoch [14], sondern trägt zum Mangel an Inanspruchnahme von fachpsychiatrisch-psychotherapeutischer Hilfe bei. Wer sich schämt, an einer schizophrenen Störung zu leiden, nimmt weniger professionelle Hilfe in Anspruch und steigt seltener in berufliche und soziale Integrationsmaßnahmen ein [14-16]. Aus solchen Überlegungen wurden neue edukative Ansätze entwickelt , die mit „normalizing“ (z. B. interaktive Erarbeitung eines gemeinsamen Arbeitsmodells zu Therapieplanung unter Rückgriff auf Beschreibung psychotischen Erlebens als Extremvariante normalpsychologischen Funktionierens) und stärkerer Partizipation des Patienten $[17,18]$ an nachhaltiger Behandlungsbereitschaft durch motivationspsychologische Ansätze (z.B. des „shared decision making“) ansetzen. Dieses Vorgehen ist beispielgebend für andere psychische Störungen.

Argument 4: Multiprofessionelle ambulante Behandlung mit Case Management bei schizophrenen Störungen übernahm eine Vorreiterrolle, indem sie bei Schizophrenie lange vor anderen Störungsbereichen in den Vordergrund rückte [19].

Die Notwendigkeit ambulanter multiprofessioneller Versorgungsteams in der gemeindenahen integrierten Versorgung wurde bei schizophrenen Störungen erkannt [20] und zum Vorbild für die Versorgung schwerer anderer psychischer Erkrankungen. Dieser Ansatz orientiert sich flexibel am individuellen Hilfebedarf. Medizinische Modelle rein symptomorientierter Versorgung werden aus intrapersonellem Störungsblickwinkel gestörter Neurobiologie in Richtung des Verständnisses von „Krankheit“ als entwicklungspsychologisch relevantem „critical life event" ausdifferenziert. Psychotherapeutisch wird die Arbeit am beschädigten Selbstkonzept erforderlich. „Krankheit“ wird als interpersonelles Geschehen in neuen Behandlungs- und Versorgungsmodellen aufgegriffen [3].

Argument 4: Das Primat „ambulant vor stationär“ und die Förderung gemeindenaher integrativer Versorgung ist nicht zuletzt am Beispiel schizophrener Störungen deutlich geworden.

Die Behandlung schizophrener Patienten in ihrer sozialen und beruflichen Alltagssituation eröffnet einen repräsentativen $\mathrm{Zu}$ griff auf symptomatische, nebenwirkungsbezogene und funktionale therapeutische Zielbereiche [21]. Hier werden folgende Ziele erreicht: Durch den Verbleib im Alltag und die Verhinderung einer Abkopplung von Behandlung und Alltag reduziert sich die (Selbst-)Stigmatisierung des Patienten. Im „Kontakt“ mit dem Alltag kann eine repräsentativere Sammlung von Problembereichen des Patienten stattfinden, sodass der Therapiefokus korrekter gesetzt und der Transfer des in Therapie und Rehabilitation Gelernten in den Alltag wesentlich verbessert wird [22].

Argument 5: Leitkrankheit unseres Fachs sind schizophrene Störungen auch insofern, als einerseits weiterentwickelte moderne Ansätze große Chancen für Einsparungen eröffnen (v. a. im statio- nären Bereich; [23]), andererseits evidenzbasierte aktuelle Versorgungskonzepte im ambulanten Bereich besonders vulnerabel für unangemessene gesundheitsökonomische Einsparungen sind.

Stationäre Aufenthalte werden unter ökonomischem Druck immer kürzer. So werden Menschen mit schizophrenen Störungen symptomatisch weniger stabilisiert und auch seltener mit geklärter sozialer oder beruflicher Integrationsperspektive entlassen. Die Anforderungen an ambulante „Nachsorgesysteme“ nehmen zu. Dies gilt für Institutsambulanzen, mobile Equipen und Niedergelassene gleichermaßen. Aber eingesparte stationäre Budgets werden fast nie in das ambulante Versorgungssystem reinvestiert, da nur selten ein einheitlich verwalteter Finanztopf besteht. Andererseits bedrohen gesundheitsökonomischer Druck und insbesondere falsche gesundheitspolitische Anreize gerade hier auch die Implementierung von „Goldstandards“ zeitgemäßer evidenzbasierter Behandlung: So sind betriebswirtschaftliche Leitungen von Kliniken nahezu stolz, wenn sich höherer betriebswirtschaftlicher Erfolg erzielen lässt durch Absenkung der Personaldecke unter die Vorgaben der Psychiatrie-Personalverordnungen, durch geringere Löhne des Personals, durch höhere „case loads“ in Institutsambulanzen von 150 Patienten je Mitarbeiter (anstelle des international üblichen „case loads“ von 40 bei „supportivem“ und 25 bei „störungsspezifischem verhaltenstherapeutischem Angebot") oder durch den vorrangigen Einsatz von Antipsychotika der ersten Generation im Bereich der Akutversorgung. Der Preis hierfür ist längerfristig hoch. Ungünstige, weil nebenwirkungsreichere Krisenmedikation führt zu negativen Behandlungserfahrungen, die später die rechtzeitige Inanspruchnahme von Hilfe in Krisen oder sogar den nachhaltigen Aufbau poststationärer Compliance insgesamt gefährdet. Hieraus erklärt sich auch (neben den Ausbildungsmängeln), warum weniger als 5\% aller Patienten mit schizophrenen Störungen überhaupt störungsspezifische Psychotherapie erhalten [24], während europäische Angehörigenverbände wie EUFAMI in ihrer Osloer Deklaration im Jahr 2000 oder in der Basler Deklaration im Jahr 2011 (www.eufami.org) schon lange die breite Implementierung evidenzbasierter Behandlung und Versorgung auf der Basis moderner wissenschaftlicher Erkenntnisse für ihre erkrankten Familienmitglieder fordern.

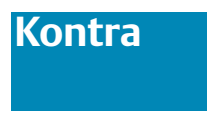

In der Geschichte der Psychiatrie gab es schon immer Kernerkrankungen, mit denen das Fach besondersassoziiert wurde. Dies traf insbesondere für die schizophrenen Psychosen zu, nachdem sich die Psychiatrie des 19. Jahrhunderts bis in die 60er-Jahre des 20. Jahrhunderts intensiv mit dem Krankheitsbild der Schizophrenie beschäftigte. Dementsprechend befasste sich die Konzeptentwicklung der Psychiatrie sehr stark mit Psychoseerkrankten und es bildeten sich viele innovative gemeindenahe Versorgungsstrukturen, die auf die besonderen Bedürfnisse dieser Patienten zugeschnitten wurden. Insofern war die Schizophrenie tatsächlich so etwas wie eine „Leitkrankheit“ der Psychiatrie. Seit einigen Jahrzehnten hat sie allerdings diese Stellung verloren.

Argument 1: Das Behandlungsspektrum in der Psychiatrie hat sich vollständig verändert.

Neuere Untersuchungen gehen davon aus, dass etwa 1\% der Bevölkerung einmal im Leben an einer Schizophrenie erkranken [25]. Die Lebenszeitprävalenzen anderer psychischer Erkrankungen sind um ein Vielfaches höher, z.B. affektive Störungen (15$20 \%)$, Angststörungen (10-15\%), Alkoholabhängigkeit (8-14\%) 
oder demenzielle Erkrankungen (ca. 7\% der über 65-Jährigen) [26]. Diese Erkrankungen haben enorme volkswirtschaftliche und gesundheitspolitische Auswirkungen und sind zunehmend in den Mittelpunkt des Faches Psychiatrie und Psychotherapie gerückt. Konsequenterweise sank der Anteil der wegen Schizophrenie, schizotypen oder wahnhaften Störungen stationär behandelten Patienten kontinuierlich. So halbierte er sich in unserer Klinik seit 1997 von ca. 17\% auf aktuell nur noch $8-9 \%$. Im selben Zeitraum verdoppelte sich der Anteil der wegen Persönlichkeitsstörungen (v.a. Borderline-Persönlichkeitsstörungen) behandelten Patienten, und auch der Anteil der Patienten mit depressiven Störungen nahm zu. Dies hat zur Folge, dass gut die Hälfte der heutzutage stationär behandelten psychiatrischen Patienten vorrangig psychotherapeutisch zu behandeln ist. Das heißt, dass psychotherapeutische Versorgungs- und Therapiekonzepte für die Behandlung dieser Patienten, die wir heute vorrangig in den Kliniken sehen, zu entwickeln sind.

Ein solches Konzept sieht an modernen Kliniken für Psychiatrie und Psychotherapie eine Binnendifferenzierung vor, die auf die Bedürfnisse der jeweiligen Krankheitsgruppe ausgerichtet ist. So werden diese Patienten auf unterschiedlichen Schwerpunktstationen mit den auf sie zugeschnittenen spezifischen Psychotherapiekonzepten behandelt, z. B.

- Patienten mit Angst- und Zwangsstörungen mit kognitiver Verhaltenstherapie (KVT) und multimodaler Therapie mit Expositionsverfahren

- Patienten mit Borderline-Persönlichkeitsstörungen mit dialektisch-behavioraler Therapie (DBT)

- Patientinnen mit Essstörungen mit kognitiv-behavioraler Therapie (CBT-E nach Fairburn)

- Patienten mit chronisch depressiven Störungen mit Cognitive Behavioral Analysis System of Psychotherapy (CPASP) und KVT

- Patienten mit psychotischen Störungen mit kognitiver Verhaltenstherapie und Psychoedukation

Hinzu kommen Spezialambulanzen im Rahmen der Psychiatrischen Institutsambulanz. Zudem wird auch der sozialpsychiatrische Versorgungsbereich umstrukturiert, um den Bedürfnissen des veränderten Versorgungsspektrums Rechnung zu tragen. Dies geschieht z.B. durch spezialisierte Wohngemeinschaften für Borderline-Persönlichkeitsstörungen und andere Patientengruppen - neben den klassischen Einrichtungen für Psychoseerkrankte.

Argument 2: Die Versorgung nichtschizophrener Patienten hat das Anforderungsprofil des Psychiaters grundlegend gewandelt. Von Leitkrankheiten medizinischer Disziplinen kann erwartet werden, dass sie das Behandlungsspektrum der jeweiligen Facharztgruppe nachhaltig prägen. Es ist aber sicher unbestritten, dass sich das Anforderungsprofil des Facharztes für Psychiatrie und Psychotherapie in den letzten 20 - 30 Jahren sehr stark geändert hat. Bestimmend ist heute ein dreidimensionales Behandlungskonzept, das die neurobiologische, sozialtherapeutische und psychotherapeutische Dimension erfasst. Dieses Konzept wurde nicht vorrangig an der Schizophrenie entwickelt, sondern v.a. die psychotherapeutische Kompetenz wurde durch die Beschäftigung mit Angst- und Zwangsstörungen, Depressionen, posttraumatischen Belastungsstörungen und anderen Erkrankungen eingebracht [27]. Die notwendigen Kompetenzen und Aufgaben der modernen Psychiater sind vielfältiger und komplexer geworden. Ihr Tätigkeitsprofil umreißt zum einen die Bereiche, die für die Weiterbildung zum Facharzt für Psychiatrie und Psychotherapie relevant sind, dient aber auch der Abgrenzung zu benachbarten Berufsgruppen. Auf europäischer Ebene wurde von einer Arbeitsgruppe der Europäischen Facharztvereinigung UEMS („Union Européenne des Médecins Spécialistes“) ein entsprechendes Profil („The Profile of a Psychiatrist“) mit dem Ziel ausgearbeitet, die heute notwendigen Kompetenzen und Aufgaben von Psychiaterinnen und Psychiatern in Europa zu beschreiben. Demnach gliedert sich deren Kompetenz- und Anforderungsprofil in folgende Bereiche: „Psychiatric Expert/Clinical Decision-Maker“, „Communicator“, „Collaborator“, „Manager“, „Health Advocate“, „Scholar“, „Professional“ [28]. Das Ziel des Psychiaters richtet sich also nicht mehr nach einer einzelnen Störung wie der Schizophrenie, sondern nach dem komplexen Anforderungsprofil in verschiedenen Arbeitsbereichen. Somit gibt es keine identitätsstiftende Leitkrankheit der Psychiatrie mehr.

Argument 3: Die klinische Grundlagenforschung an sog. „neurotischen“ Erkrankungen hat das biopsychosoziale Krankheitsmodell wesentlich geprägt.

Während man früher der Meinung war, dass bei den sog. „endogenen Psychosen“ vorrangig pharmakotherapeutische Ansätze indiziert sind und Psychotherapie lediglich eine untergeordnete Rolle spielt, wohingegen die Psychotherapie bei den sog. „neurotischen Erkrankungen“ Behandlungsmethode der Wahl ist, hat die weitere Forschung gezeigt, dass eine solche Dichotomisierung nicht aufrechtzuerhalten ist. Psychotherapie spielt neben soziotherapeutischen und pharmakotherapeutischen Interventionen bei der Psychosebehandlung eine Rolle, wohingegen beispielsweise Angststörungen und Zwangsstörungen erfolgreich pharmakotherapeutisch behandelt werden können [27]. Neben diesen Ergebnissen der klinischen Evaluationsforschung hat auch die klinische Grundlagenforschung gerade an nichtpsychotischen Erkrankungen gezeigt, dass Psychotherapie neurobiologische Parameter verändert. Eines der wichtigsten Störungsbilder in diesem Zusammenhang stellt die Zwangsstörung dar, bei der bereits in den 90er-Jahren gezeigt werden konnte, dass Psychotherapie bei Respondern auf kognitive Verhaltenstherapie die Aktivität des fronto-striato-thalamischen Regelkreises modifiziert [29, 30]. Unsere Arbeitsgruppe konnte zeigen, dass erfolgreiche kognitive Verhaltenstherapie neurobiologische Parameter des fronto-striato-thalamischen Regelkreises verändert und diese wiederum Prädiktoren für den Behandlungserfolg von Psychotherapie darstellen [31]. Dass heute Pharmako- und Psychotherapie keine Gegensätze, sondern sich ergänzende Behandlungsmöglichkeiten darstellen, ist nicht zuletzt durch Evaluationsforschung und neurowissenschaftliche klinische Grundlagenforschung an nichtpsychotischen Erkrankungen entwickelt worden. Diese Forschungsergebnisse haben unser Konzept von Psychiatrie wesentlich verändert.

Argument 4: Die Psychiatrie beschäftigt sich wissenschaftlich vorrangig mit nichtschizophrenen Erkrankungen.

Auch im Bereich der psychiatrischen Forschung ist die Psychose längst nicht mehr die führende Erkrankung, die erforscht wird, da sich auch hier das Spektrum deutlich geweitet hat. Anstatt ganze Krankheitsentitäten wie z.B. „Schizophrenie“ zu beforschen, werden zunehmend einzelne Funktionen und ihre Interaktionen untereinander beforscht, wie z.B. Aggression, Impulskontrolle, Emotionen oder Kognitionen (Roadmap für das Gesundheitsforschungsprogramm der Bundesregierung [32]). Eine eigene Auswertung aller DFG-geförderten Studien im Bereich Psychotherapie/Psychoedukation (zuständig sind hier die Fachkollegien Psychologie und Neurowissenschaft) im Zeitraum 1/ 2005 - 8/2010 ergab folgende Verteilung: Hinsichtlich der untersuchten Verfahren dominierte die kognitive VT $(n=7)$, gefolgt von Extinktion/Exposition ( $n=2)$, Psychoedukation $(n=2)$, CBASP 
$(n=1)$, dialektisch-kognitive Traumatherapie $(n=1)$. Die in den Studien/Projekten im Mittelpunkt stehenden Störungsbilder/Erkrankungen waren die Depression $(n=6)$, gefolgt von PTSD $(n=$ $2)$, soziale Phobie $(n=2)$, Schizophrenie $(n=2)$, koronare Herzerkrankung $(n=2)$, Paruresis $(n=1)$, chronischer Schmerz $(n=1)$, Bulimia nervosa $(n=1)$, bipolare Störungen $(n=1)$.

\section{Fazit und Ausblick}

Die Schizophrenie war einst v.a. deswegen Leiterkrankung der Psychiatrie, weil sich in ihren großen Anstalten und Kliniken v.a. Psychoseerkrankte aufhielten. Mit dem Wandel des Diagnosespektrums veränderten sich auch die Anforderungskompetenzen an den Psychiater und mit ihnen auch das gesamte Bild der Psychiatrie.

Nach Hochrechnung der Weltgesundheitsorganisation WHO werden im Jahr 2030 die psychischen Erkrankungen für die Hauptlast der durch Behinderung bzw. Arbeitsunfähigkeit belasteten Lebensjahre (DALY's = Disability Adjusted Life Years) der Bevölkerung in den Industrieländern verantwortlich sein. Die Prognose nennt hier an erster Stelle die unipolaren Depressionen, gefolgt von der Alkoholabhängigkeit. Mit Demenzen, Schizophrenie und bipolaren Störungen werden 3 weitere psychische Erkrankungen auf den Rängen 4-6 gelistet (Burden of Disease Study [33]). Ob allerdings die immer populärer werdende Meinung, dass die „Volkskrankheit“ Depression auch als Leitkrankheit des 21. Jahrhunderts [34] anzusehen ist, zutrifft, steht auf einem anderen Blatt. Zielt doch diese Zuschreibung nicht nur auf das Selbstverständnis und die Orientierung innerhalb eines medizinischen Faches, sondern auf eine größere, gesamtgesellschaftliche Dimension. Es wäre interessant zu beobachten, ob die Anerkennung und Aufmerksamkeit, die den psychischen Erkrankungen insgesamt und der Depression als epochale Leitkrankheit im Besonderen widerfährt, einhergeht mit der Beförderung der Psychiatrie zur „medizinischen Wissenschaft des 21. Jahrhunderts“.

\section{Literatur}

1 van Os J, Kenis G, Rutten BP. The environment and schizophrenia. Nature 2010; 468: $203-212$

2 Vauth R, Cavelti M, Schieting S et al. Recovery bei schizophrenen Störungen: Ergebnis und Prozess im Fokus moderner Behandlung. Schizophrenie Mitteilungen der gfts 2011; 27: 50 - 57

3 Vauth $R$. Psychologische Mechanismen bei der Entwicklung von Depression und ihre Implikationen für psychotherapeutische Ansatzpunkte bei schizophrenen Störungen. neuro aktuell (im Druck)

4 Vauth $R$. Die umfassende Behandlung schizophrener Störungen als klinische Herausforderung. In: Strauß B, Hohagen F, Caspar F, Hrsg. Lehrbuch Psychotherapie. Teilband 1. Göttingen: Hogrefe; 2007: 611 -646

5 Kumari V, Peters ER, Fannon $D$ et al. Dorsolateral prefrontal cortex activity predicts responsiveness to cognitive-behavioral therapy in schizophrenia. Biol Psychiatry 2009; 66: 594-602

6 Linden $D E$ et al. How psychotherapy changes the brain - the contribution of functional neuroimaging [Review]. Molecular psychiatry 2006; 11: $528-538$

7 Dickerson FB, Lehman AF. Evidence-based psychotherapy for schizophrenia: 2011 update. J Nerv Ment Dis 2011; 199: 520 - 526

8 Garety PA, Bebbington B, Fowler D et al. Implications for neurobiological research of cognitive models of psychosis: a theoretical paper. Psychological Medicine 2007; 37: 1377-1391

9 Kuipers E, Garety P, Fowler D et al. Cognitive, emotional, and social processes in psychosis: refining cognitive behavioral therapy for persistent positive symptoms. Schizophr Bull 2006; 32 (Suppl. 01): 24-S31

10 Rathod S, Phiri P, Kingdon D. Cognitive behavioral therapy for schizophrenia. Psychiatr Clin North Am 2010; 33: 527-536

11 Tarrier $N$. Cognitive behavior therapy for schizophrenia and psychosis: current status and future directions. Clin Schizophr Relat Psychoses 2010; $4: 176-184$
12 Lysaker PH, Tunze C, Yanos PT et al. Relationships between stereotyped beliefs about mental illness, discrimination experiences, and distressed mood over 1 year among persons with schizophrenia enrolled in rehabilitation. Soc Psychiatry Psychiatr Epidemiol 2011: May 21 [Epub ahead of print]

13 Cavelti M, Kvrgic S, Beck EM et al. Self-stigma and its relationship with insight, demoralization, and clinical outcome among people with schizophrenia spectrum disorders. Compr Psychiatry 2011: Sep 26 [Epub ahead of print]

14 Vauth R, Kleim B, Wirtz M et al. Self-efficacy and empowerment as outcomes of self-stigmatizing and coping in schizophrenia. Psychiatry Research 2007; 150: $71-80$

15 Beck EM, Cavelti M, Kvrgic S et al. Are we addressing the "right stuff" to enhance adherence in schizophrenia? Understanding the role of insight and attitudes towards medication Schizophr Res 2012; 132 : 42-49

16 Corrigan PW, Rafacz J, Rusch $N$. Examining a progressive model of selfstigma and its impact on people with serious mental illness. Psychiatry Res 2001; 189: 339 - 343

17 Hamann J, Mendel R, Cohen R et al. Psychiatrists' use of shared decision making in the treatment of schizophrenia: patient characteristics and decision topics. Psychiatr Serv 2009; 60: 1107-1112

18 Kreyenbuhl J, Nossel IR, Dixon LB. Disengagement from mental health treatment among individuals with schizophrenia and strategies for facilitating connections to care: a review of the literature. Schizophrenia Bulletin 2009; 35: 696-703

19 Miklowitz DJ, Otto MW, Frank E et al. Intensive psychosocial intervention enhances functioning in patients with bipolar depression: results from a 9-month randomized controlled trial. American Journal of Psychiatry 2007; 164: $1340-1347$

20 Vauth $R$. Psychotherapie bei schizophrenen Störungen auf der Grundlage modernen Case Managements. In: Falkai P, Pajonc FG, eds. Langzeittherapie der Schizophrenie. Bremen: Unimed; 2004: 84-97

21 Dixon LB, Dickerson F, Bellack AS et al. The 2009 schizophrenia PORT psychosocial treatment recommendations and summary statements. Schizophr Bull 2010; 36: 48-70

22 Vauth $R$, Corrigan PW, Clauss $M$ et al. Cognitive strategies versus selfmanagement skills as adjunct to vocational rehabilitation. Schizophr Bull 2005; 31: 55-66

23 Salize HJ, McCabe R, Bullenkamp J et al. Cost of treatment of schizophrenia in six European countries. Schizophr Res 2009; 111: 70-77

24 Puschner B, Vauth R, Jacobi F et al. Evidence basis of psychotherapy for schizophrenia patients in Germany. Nervenarzt 2006; 77: 1301 -1302, 1304-1309

25 Wobrock T, Pajonk FG, Falkai P. Schizophrenie. Teil 1: Epidemiologie. Ätiopathogenese, Symptomatologie. Fortschr Neurol Psychiat 2004; 72: $98-113$

26 Meyer C, Rumpf H-J, Hapke $U$ et al. Lebenszeitprävalenz psychischer Störungen in der erwachsenen Allgemeinbevölkerung. Ergebnisse der TACOS-Studie. Nervenarzt 2000; 71: 535-542

27 Lotz-Rambaldi W, Hohagen F. Entwicklung der Psychotherapie im Fachgebiet Psychiatrie und Psychotherapie. Erfolgreiche Einbindung. NeuroTransmitter 2009; 2 (Sonderheft): 6-12

28 Hohagen $F$. The profile of a psychiatrist. Nervenarzt 2006; 77: 748 - 750

29 Baxter LR Jr, Schwartz JM, Bergman KS et al. Caudate glucose metabolic rate changes with both drug and behavior therapy for obsessive-compulsive disorder. Arch Gen Psychiatry 1992; 49: 681 - 689

30 Schwartz JM, Stoessel PW, Baxter LR Jr et al. Systematic changes in cerebral glucose metabolic rate after successful behavior modification treatment of obsessive-compulsive disorder. Arch Gen Psychiatry 1996; 53: 109-113

31 Zurowski B, Kordon A, Weber-Fahr $W$ et al. Relevance of orbitofronta neurochemistry for the outcome of cognitive-behavioural therapy in patients with obsessive-compulsive disorder. Eur Arch Psychiatry Clin Neurosci 2012: (in press) DOI: 10.1055/s-0029-124558010.1007/ s00406-012-0304-0

32 Gesundheitsforschungsrat (GFR) des Bundesministeriums für Bildung und Forschung (BMBF). Roadmap für das Gesundheitsforschungsprogramm der Bundesregierung. 2007

33 Mathers $C D$, Loncar $D$. Updated projections of global mortality and burden of disease, 2002-2030: Data sources, methods and results. Public Library of Science Medicine 2006; 3: e442

34 Volk der Erschöpften. DER SPIEGEL 2001; 4: 114-122 